\title{
ICF-based Classification to Bridge the Gap Between End-Users and AAL Solutions
}

\author{
Francesca Fracasso, Gabriella Cortellessa, Karen Coan, Gilbert Regan, Pierre \\ Rossel, Alessandro Umbrico, Amedeo Cesta
}

\begin{abstract}
MAESTRO (Sustainable Reference Framework evaluating equipment and services for seniors) is a web-based ICT multi modal platform providing a broad range of services and benefits in the domain of monitoring and self-monitoring systems for well-being and health-related information acquisition. Specifically, MAESTRO aims at realizing an innovative framework capable of facilitating the interaction and communication between producers and consumers at different levels, taking into account needs of end-users and features of products. This paper provides an overview of the key concepts and capabilities of MAESTRO focusing on the use of the International Classification of Functioning, Disability and Health (ICF) to make profiles of end-users and discover and rank products that meet their health-related needs. Also, this paper shows the results of a study aimed at evaluating the classification capabilities of MAESTRO and the integrated ICF-based taxonomy with respect to products and services developed within AAL Projects. The evaluation points out the health-related needs of end-users AAL projects focus the most as well as some limits and possible enhancements of the integrated ICF-based taxonomy.
\end{abstract}

Amedeo Cesta, Gabriella Cortellessa, Francesca Fracasso and Alessandro Umbrico National Research Council of Italy, Institute of Cognitive Sciences and Technologies (ISTC-CNR),

Via S. Martino della Battaglia, 44, 00185, Rome, Italy,

e-mail: \{name.surname\}@istc.cnr.it

Karen Coan, Gilbert Regan

Dundalk Institute of Technology (DkIT)

e-mail: \{name.surname\} @dkit.ie

Pierre Rossel

Coherent Streams Srl

Avenue des Baumettes, 5, 1020, Renens, Switzerland

e-mail: pr@coherentstreams.com 


\section{Introduction}

There has been a growing trend towards self-surveillance in the last 30 years. Only recently, however, technologies, both as stand-alone systems, with the sensor-level components, and the related "connected object" value chain, with its cabled and/or wireless communications means, along with big data acquisition and management capabilities, have really been delivering the kind of performance and service corresponding to expectations.

What can be observed is a proliferation of announcements, in particular in an expanding market comprising wristbands, smart watches, smartphone applications and to a lesser extent smart glasses, shoes and clothes, with connections and data acquisition at home, in cars or other venues. Many sectors or markets are supporting this trend, in particular sports, wellness and defense/security activities. However, while such tools tend not to be designed for or to target older adults as end users, this group can benefit enormously from such self-monitoring technologies.

In this fast-proliferating context, the clues and control references for adequate orientation of the consumer are lacking and the result is a media-supported image of a fantastic potential for follow-up of one's activities and even some vital parameters, but with often no verified nor legitimised/verified less certified criteria (there are exceptions of course) regarding such key dimensions as performance, reliability, user-friendliness, acceptability, potential for delivering medically ascertained data, privacy issues, etc.

This confusing and probably in many cases misleading landscape, is even more problematic for the elderly, often non fully digitally literate and in any case not always in the capacity to know, understand and distinguish the key elements of the underlying promises associated with these technologies. There is therefore at the same time a claim to give access to truly helpful systems for well-being and healthrelated monitoring capabilities, but most of the time as an emerging market not accompanied by the kind of references and metrics which could establish the basis for an effective user centered affordance of these new means.

In this scenario, MAESTRO (Sustainable Reference Framework evaluating equipment and services for seniors) is a web-based ICT multi modal platform providing a broad range of services and benefits in the domain of monitoring and selfmonitoring systems for well-being and health-related information acquisition. It tackles specific user groups with clearly identified service scenarios, the overall objective is to build upon of this results a reference framework, so as to bring to a variety of users enhanced orientation and evaluation capabilities based on user profile, selected solution or service, as well as decision-making readiness for developments and investment.

MAESTRO is an AAL (Active Assisted Living) programme project that started in October 2015 until December 2018. The partnership is composed of members from Luxembourg (lead partner), Italy, Switzerland and Ireland. It aims at buildingup, testing and disseminating in the broad European context, through an open platform, a dynamic Reference Framework (RF) concerning monitoring and selfmonitoring systems, connected or not. The Reference Framework MAESTRO in- 
tends to construct does not mean to define ideal tools and forms of usage but on the contrary needs to be built upon existing efforts and experiences, in particular best practises and corresponding functionalities and services (see further), to specify the options and criteria supporting these exemplary configurations.

This paper provides an overall description of the main concepts of MAESTRO, focusing on the classification and recommendation capabilities of the platform.

\section{The MAESTRO Project}

MAESTRO aims at realizing a set of services that allow seniors and/or caregivers to keep in contact with product manufacturers. Dedicated services allow seniors/caregivers to search and buy products suited for their specific needs, and manufacturer to sell their own products.

The challenging objective of MAESTRO is to realize a framework capable of facilitating the interaction and communication between producers and consumers at different levels, taking into account needs of end-users and features of products. On the one hand, MAESTRO proposes a clear taxonomy to characterize end-users according to their health-related needs, and products according to health-related needs they can address/satisfy. On the other, MAESTRO proposes a "standard" to evaluate/assess products according to a number of well-defined metrics and qualities. Assessments leverage direct user experience to characterize several aspects of products and provide manufacturers with useful feedbacks to improve their solutions.

Therefore, MAESTRO realizes a twofold value chain: (i) a Business-to-Business (B2B) value chain which aims at supporting manufacturers in improving the quality of their products; (ii) a Business-to-Consumers (B2C) value chain which aims at facilitating manufacturers in reaching their potential consumers as well as consumers in findings products suited for their specific needs.

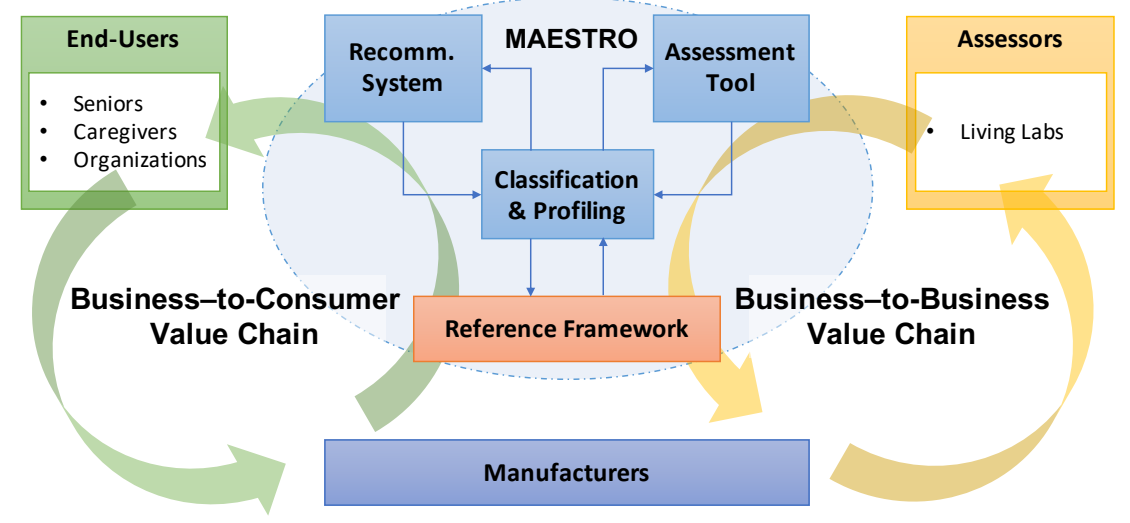

Fig. 1 Overview of the MAESTRO framework 
Figure 1 shows a conceptual view of MAESTRO showing, the involved stakeholders and their relationships.

As can be seen, three types of stakeholder are involved in MAESTRO. End-users represent seniors, caregiver or caregiving organizations that want to find products that are suited to their specific needs. They are interested in using MAESTRO because it supports them in finding high quality products that are suited for their needs.

Manufacturers represent companies that produce one or more types of product and that want to reach a large number of highly interested consumers. They are interested in using MAESTRO because it represents a well-structured channels with potential clients but also because it defines a clear "evaluation standard" which provides them with structured feedbacks from the market.

Assessors represent a particular type of stakeholder that support MAESTRO providing assessments of products that comply with the proposed "evaluation standard". These stakeholders play a crucial role in the platform. They are in charge of making assessments and feedbacks that characterize products with respect to different quality dimensions.

The core element of MAESTRO is the Reference Framework (RF) which consists of concepts, properties and taxonomies that define the proposed "MAESTRO standard". RF is the basic element used to classify manufacturers' products according to different dimensions and profile end-users according to their specific health-related needs. Two main services rely on these classification and profiling capabilities and the related $\mathrm{RF}$.

The Recommendation System service is in charge of analyzing health-related profiles of end-users and products to dynamically match supply and demand. There are a huge number of products available through MAESTRO platform. Each product has its own features and qualities. The recommendation system service supports end-users by ranking available products according to their specific profiles. This service is crucial to realize the B2C value chaing of MAESTRO. The classification and profiling capabilities allow MAESTRO to support end-users showing/recommending products whose profiles are closer to their specific needs.

The Assessment Tool service instead is in charge of dynamically selecting the evaluation criteria assessors must take into account to assess products. Specifically, it analyzes the metrics and qualities that, according to RF and to the specific profile of a product, are relevant for evaluation. This allows assessors to make evaluations that comply with the "MAESTRO standard" and that enable (fair) comparisons among similar products (e.g, products targeted for similar health-related needs). The assessment capabilities allow MAESTRO to guide assessors in the evaluation process whose outcomes provide therefore valuable feedbacks to manufacturers to improve products.

This paper focuses on the recommendation services of MAESTRO and therefore next section provides additional details on the structure of the RF. Specifically it characterizes the set of information used to classify products and profile end-users according to their specific needs. 


\section{The ICF-based Recommendation System}

The key objective of the Recommendation System is to identify health-related needs of end-users and find out the products that best fit these needs. To this aim, MAESTRO must be endowed with the capabilities needed to internally represent information about needs of end-users and "connect" these needs to products that can satisfy them.

Health-related needs represent a complex knowledge which requires the system to deal with and properly organize a large and heterogeneous amount of information. Namely, it is necessary to characterize needs and behaviors of end-users according to a well-defined semantics to realize the elicited recommendation capabilities.

The MAESTRO Reference Framework integrates a dedicated representation of the International Classification of Functioning, Disability and Health (ICF) which has been proposed by the World Health Organization (WHO) [Organization et al., 2001]. The ICF defines a complete semantics for representing physical and cognitive capabilities of persons by taking into account different perspectives and dimensions. Therefore, it provides the core concepts needed to build profiles of end-users and products. These profiles/classifications are then used by the recommendation system to support end-users.

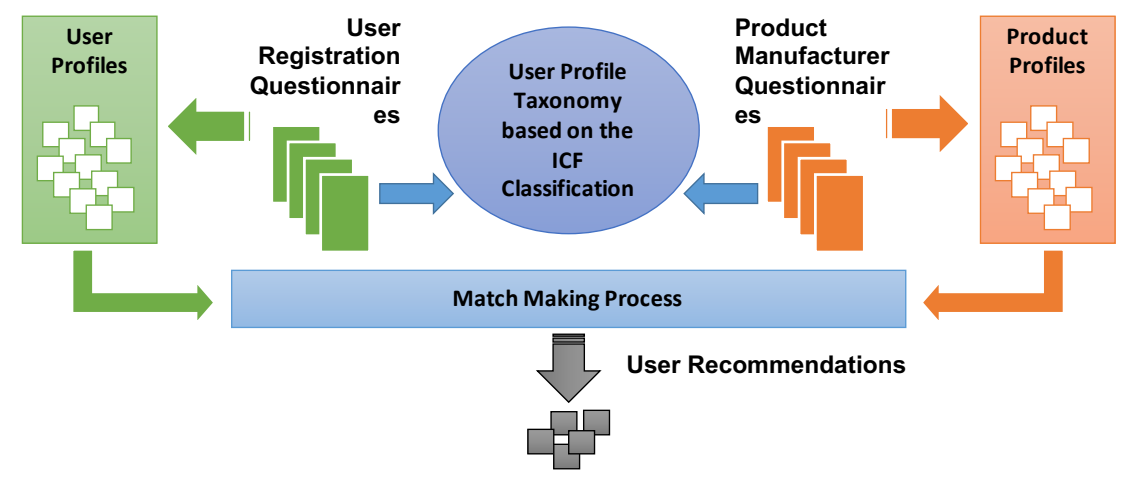

Fig. 2 A conceptual overview of the MAESTRO recommendation system

Figure 2 shows a conceptual representation of the recommendation system service. It shows how recommendations are generated starting from end-users and product profiles. Both of them built on top of the integrated ICF taxonomy.

End-user profiles are built by means of questionnaires that are filled at registration time. Similarly, product profiles are built by means of other dedicated questionnaires that are filled when manufacturers register them into MAESTRO. These questionnaires consist of a number of questions that allow the system to characterize end-users and products with respect to the internal representation of the ICF taxonomy. Namely, each question characterizes a user or a product with respect to a specific perspective and dimension of the ICF. When completed, the resulting pro- 
files completely characterize health related needs of end-users and health-related needs satisfied by products.

These profiles are then analyzed by a match making process which is in charge of actually generating product recommendation for a particular end-user. The match making process takes as input the profile of an end-user interacting with MAESTRO. According to this profile, it analyzes the registered profiles of products and search for those that are relevant with respect to the profile of the interacting enduser. These products represent the outcome of the recommendation system service and are shown to the end-user.

\subsection{Why the ICF Taxonomy}

The International Classification of Functioning, Disabilities and Health (ICF) aims at organizing and documenting information on functioning and disability. The framework proposes the interpretation of functioning as a dynamic interaction among the health condition of a person, environmental factors and personal factors. ICF proposes a standard language and semantics for defining, measuring and classifying disability. It is worth observing that functioning and disability denote respectively the positive and negative aspects of functioning from a biological, individual and social perspective. Therefore, it pursues a multi-perspective, biopsychosocial approach whose outcome is a multi-dimensional model. This multi-dimensional view is well-suited for the objectives of MAESTRO.

ICF defines a scientific, operational basis to describe, understand and study health and health-related states. Broadly speaking, the classification is organized into two parts. A part deals with functioning and disabilities while the other part deals with contextual factors. These two parts are then further organized into two components. The components body functions and body structure belong to the part concerning functioning and disabilities. The components environmental factors and personal factors belong to the part concerning contextual factors.

Figure 3 provides a graphical representation of ICF components and their interactions. The key idea underneath ICF is that the functioning of an individual in a specific domain reflects an interaction between the health condition and the contextual in terms of environmental and personal factors. Each ICF component consists of multiple domains, and each domain consists of categories that are the entities of the classification. The conceptual framework thus defines a common language and the high-level structures of the classification that enable specific description and quantification.

MAESTRO integrates and adapts this framework to define a semantics for representing and reasoning about health-related needs of end-users and products. The integration of ICF particularly focuses on the part concerning functioning and disabilities and specifically the components body functions and structure, and activities and participation. Therefore an ICF-based taxonomy has been defined by leveraging OWL [Bechhofer, 2009] which represents a standard semantic lan- 


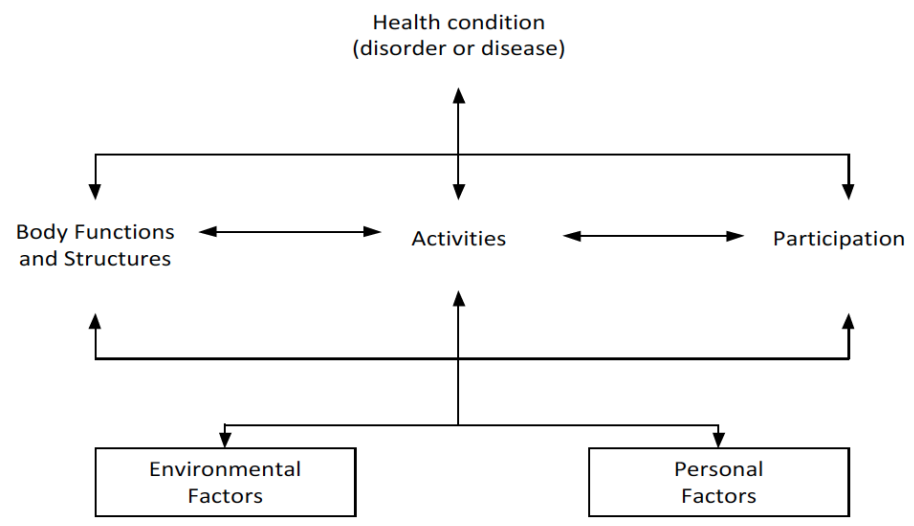

Fig. 3 An overview of the ICF model proposed in [Organization et al., 2001]

guage. The defined taxonomy relies on a foundation ontology called DOLCE [Gangemi et al., 2002] which provides a clear and well-defined semantics for basic concepts like e.g., properties, qualities and capabilities/functions. Specifically, MAESTRO leverages a simplified subset of DOLCE called DUL (DOLCE Ultra Light) ${ }^{1}$ whose expressiveness is sufficient for the specific objectives of the project.

Each class of the integrated ICF taxonomy characterizes a specific aspect of the physical and cognitive capabilities of a person. The level of each capability is measured leveraging the class DUL: Quality of DUL ontology. Specifically, each capability composing the profile of a person can be associated with a low, regular and high values, where low, regular and high are constant individuals of the class DUL: Quality. A low level of a physical or cognitive capability means that a person has a "low functioning" of that capability which represents a "disability" in the ICF sense. Conversely, a high level of a physical or cognitive capability means that a person has a "high functioning" of that capability which represents a "functionality" a person can perfectly use.

\subsection{Body Functions and Structures}

The ICF component body functions and structures concerns aspects of physiology and anatomy. The body is an integral part of human functioning and the biopsychosocial model considers it in interaction with other components. More specifically, body functions are the physiological aspects of body systems, while structures are the anatomical support. For example, sight is a function while the eye is a structure. Figure 4 shows an excerpt of the integrated ICF classification. MAESTRO focuses on the body function component of ICF which provides a representation for

\footnotetext{
${ }^{1}$ http://www.loa.istc.cnr.it/ontologies/DUL.owl
} 
many aspects that are relevant to characterize the assistive services a person needs within his/her daily-home living.

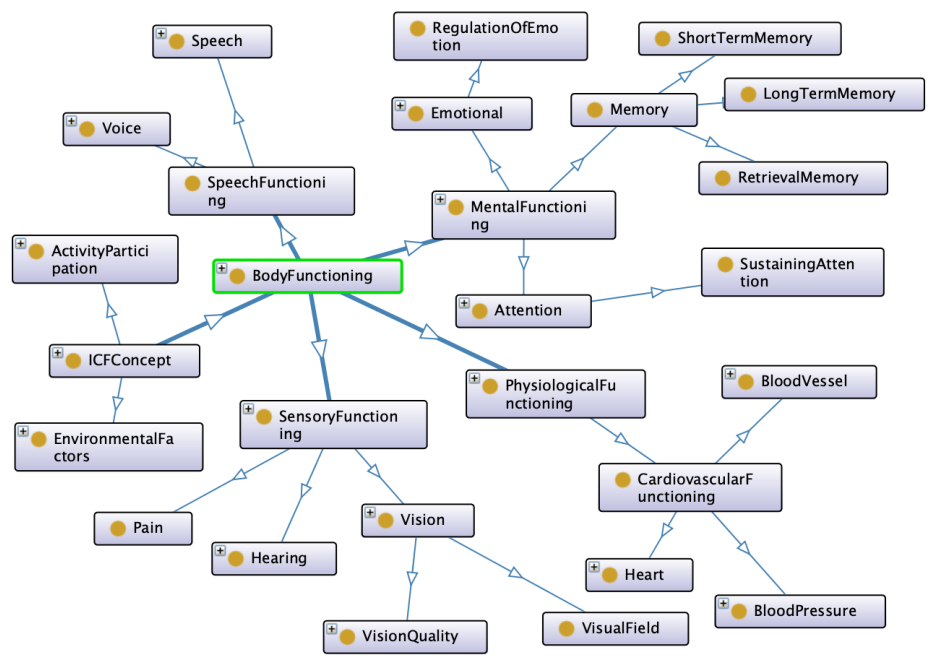

Fig. 4 An excerpt of the integrated body functions and structures ICF category

For example the class MentalFunctioning allows MAESTRO to model capabilities concerning the use of mental faculties ranging from the capability of maintaining the attention on some subject (the class SustainingAttention) to the capabilities of maintaining long term or short term memory (respectively the class LongTermMemory and the class ShortTermMemory). The class PhysiologicalFunctioning allows MAESTRO to model capabilities concerning the status of physiological parameters of a person like e.g., blood pressure (the class BloodPressure) or the heart (the class Heart and related subclasses

not shown in Figure 4 for the sake of simplicity). Other interesting elements of the ICF that allow MAESTRO to characterize the interaction capabilities of a person are the class SensoryFunctioning and the class SpeechFunctioning. In particular the class SensoryFunctioning and related subclasses like e.g., Hearing or Vision allow MAESTRO to model the capability of sensing the environment and receiving signals and information.

\subsection{Activity and Participation}

The ICF component activity and participation concerns actions and tasks executed by individuals (i.e., activities) and the involvement in social life situations (i.e., participation). This category covers all aspects of life, from basic actions such as walking and moving to complex and socially collaborative situations such as interacting 
with others or participating in school or in community life. Figure 5 shows an excerpt of the integrated ICF classification. In this case MAESTRO focuses mainly on the categories that characterize the autonomy of a person in performing his/her daily-routine activities.

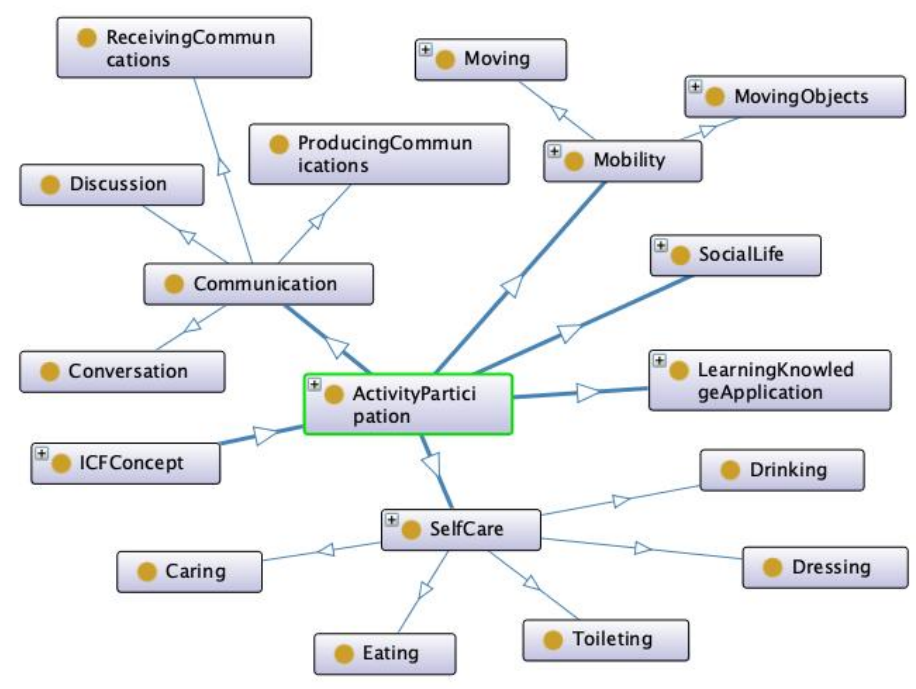

Fig. 5 An excerpt of the integrated activity and participation ICF category

For example the class Communicat ion allows MAESTRO to model capability of performing complex communication activities that range from the capability of sustaining discussions (the class Discussion) to the capability of receiving complex communications or producing complex communications (respectively the class ReceivingCommunications and the class ProducingCommunications). The class SelfCare allows MAESTRO to model the capability of a person of autonomously perform self-care related tasks like e.g., the capability of eating autonomously (the class Eating), the capability of dressing autonomously (the class Dressing) or the capability of drinking autonomously (the class Drinking). Another interesting element of the ICF which allows MAESTRO to characterize the capability of a person of autonomously and safely interacting with the environment is the class Mobility. In particular, the class Moving and related subclasses like e.g., Walking, Jumping or Running (not shown in Figure ?? for the sake of simplicity) allow MAESTRO to model the capability of moving within an environment with different modalities. 


\section{MAESTRO as a Mean to Classify AAL Projects}

A pilot of the project has been performed with the aim of investigating the feasibility of applying the MAESTRO profiling framework to classify AAL projects. AAL projects are supposed to develop complex systems to support the integration of health ageing products and providing services useful both for primary and secondary users. For this reason, investigating the efficacy of our taxonomy in their categorization could provide valuable insights about its capacity to cover as many AAL dimensions as possible.

A systematic procedure has been followed for retrieving all the available information for each project. The AAL portal ${ }^{2}$ served as starting point, since it is considered as the more reliable database on the existing projects. Projects belonging to call 2017 and previous ones have been considered in the analysis (more recent projects have not been considered since not enough information exists yet on them).

The AAL programme funded more than 220 projects since 2008. In this investigation, and as said above, we focused our attention on those funded till 2017, the more recent projects not providing sufficient information considering their state of progress. In fact, we considered 198 projects funded through yearly calls from 2008 to 2017.

Therefore, starting from the AAL portal, the information we were looking for has been retrieved from the official website of each project, and from any other type of dissemination materials available. Additionally, scientific production such as scientific papers, conference presentations and workshop participations have been also used as sources

\subsection{Overview of Classification Results}

The MAESTRO taxonomy has been applied for categorizing the AAL projects in the attempt of validating it through a number of AAL existing applications. Out of 198 funded projects, 73 could not be properly profiled through the proposed taxonomy because of insufficient available information. More specifically, not enough scientific data have been found, and in some cases the prohects' official websites were not active anymore. Although some projects could not be profiled, interesting findings emerged from this analysis.

First of all, it appeared that the major part of the projects addressed needs of older population in general, with not specific target. Some exceptions were related to the projects funded through the call 2016, which was specifically dedicated to Dementia patients ("Living well with Dementia"), and the target end-users were patients with pathological cognitive decline and/or their caregivers. Other examples can be found among other projects. For example, the VUK project specifically addressed blind people, and SmartBEAT was dedicated to heart failure patients.

\footnotetext{
${ }^{2} \mathrm{http}: / /$ www.aal-europe.eu/projects
} 
While the major part of the projects foresaw some kind of "domestic support" where ICT solutions were supposed to be deployed in the indoor environment, a number of projects aimed instead to provide support in other fields of active ageing. For example, SoulMate project's goal was to support older people in travelling, both in organizational phase by managing the trip and facilitating encounters with companions, and by supporting the trip itself. Moreover, CARELINK aims at managing the wandering problem on patients with dementia. Other attempts have made in order to foster contact between older people and caregiving realities, and their work. For example, iCareCoops was supposed to develop a new way of promoting and supporting elderly care cooperatives as a model to organize elderly care in an efficient way, as well as SpONSOR, which aimed at developing, testing and implementing an ICT platform that facilitates the posting, browsing and exchange of key information between competence-offering seniors and search-based requests, from competence-demanding organizations from the public, private and voluntary sectors. In fact, a specific call (Call 6) was supposed to fund projects proposing "ICT-based Solutions for Supporting Occupation in Life of Older Adults".

While the major part of projects addressed at first the older persons' needs and just indirectly those of caregivers, some proposals came also focusing on supporting caregivers directly. For example, the SUCCESS project provides an innovative mobile training application to support formal and informal caregivers to appropriately interact with people with dementia. As for the POSTHCARD platform, which is an engaging simulation were caregivers can practice daily life situations with Alzheimer patient

Beside these, there are some different projects like MAESTRO itself, which proposed not to design and/or develop new solutions, but a sort of "meta-solution" capable to support the whole range of users in orienting and exploiting the AAL offers, and to validate the efficacy of these offers. Another example of these "metasolution" can be I-evAALution which integrates solutions that have been developed in previous AAL-projects in order to combine all functions in one solution providing support covering the needs of the involved end-users.

Figure 6 shows a summary of the classification results. Among all the dimensions considered within the MAESTRO framework, it resulted that support to mental and emotional wellbeing, social life and health monitoring are those mostly addressed by the AAL project. On the contrary, it emerged how specific needs on sensory difficulties were somehow neglected by the developed AAL solutions with the exception of few cases which focus on specific needs (VUK for blindness).

\subsection{A Closer View to Following the ICF Dimensions}

Figure 6 shows an aggregated view of the results taking into account abstract categories of the ICF taxonomy. Interesting results can be found going deeper into the single dimensions of the ICF taxonomy considered in Figure 6 to see the specific aspects addressed by AAL projects. 


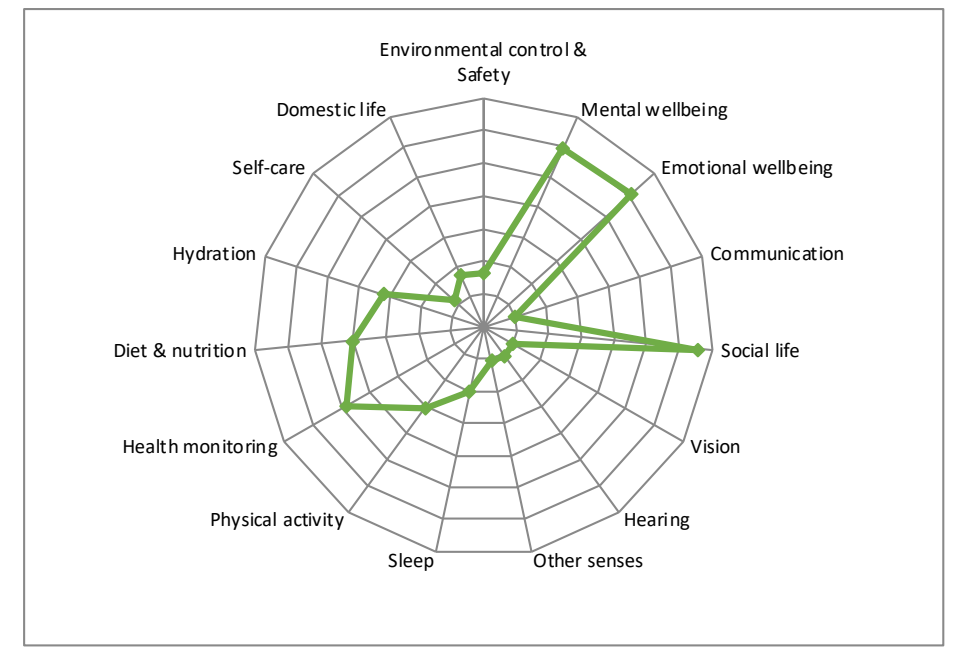

Fig. 6 Classification results of AAL proejcts with respect to the dimensions of the ICF taxonomy

Considering the dimension of mental wellbeing, the aspects mostly addressed are those regarding memory support and temporal orientation. For the dimension of emotional wellbeing, it clearly emerged how in AAL projects, the main concerns is towards designing and developing ICT solutions able to mitigate negative mood which can be overwhelming in the ageing population. Promoting solutions to foster good feelings of positive mood, i.e., being happy and content, and calm and relax, was the goal of these projects.

Social life support seems to be mostly oriented in maintaining existing relationships between older people and beloved ones, and between older people and caregivers, both formal and informal. Additionally, it seems that AAL solutions developed within the AAL program are intended to support the involvement of ageing people in the community social life.

Health monitoring emerged to be a crucial aspect in designing AAL technological solutions. Although a number of aspects have been neglected within the MAESTRO framework, it emerged the importance e of AAL community to make efforts in ensuring a reliable and effective monitoring of people health, especially with respect to parameters linked to chronic diseases of cardiorespiratory system and diabetes.

Figure 7 below shows the aggregated results divided by the specific ICF dimensions considered. 


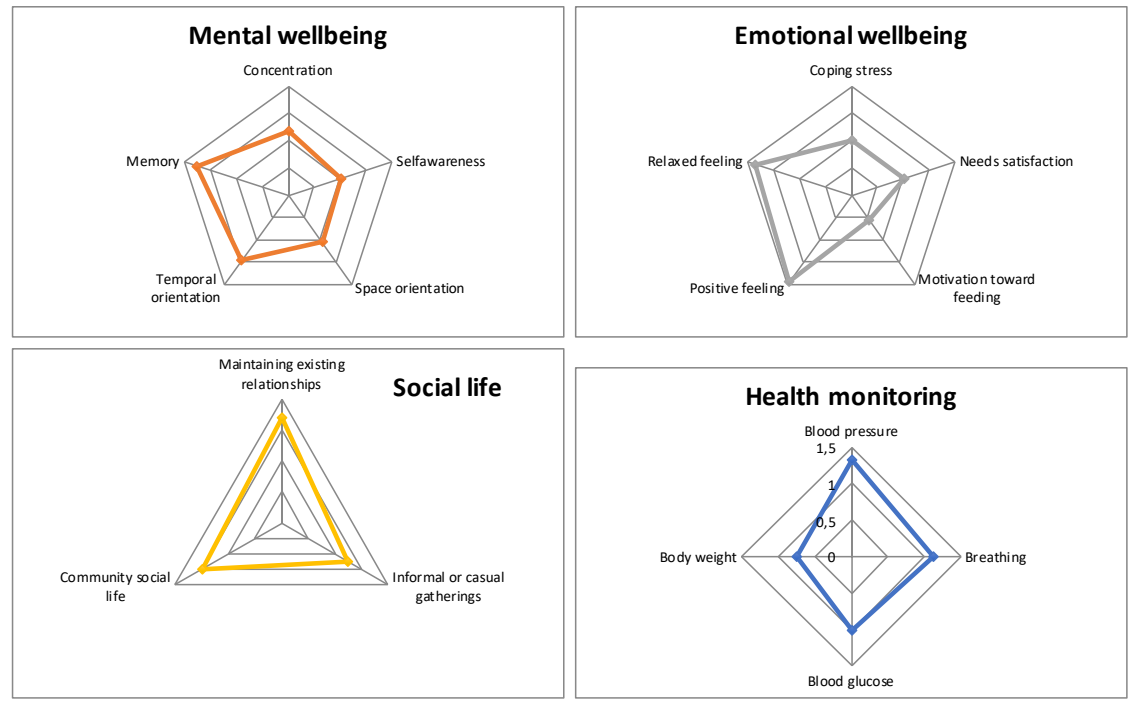

Fig. 7 Aggregated results on four different dimensions of the ICF taxonomy

\subsection{Considerations on the Classification Capabilities of MAESTRO}

The MAESTRO taxonomy was proven to be a valuable categorization framework for systems functions and services provided by AAL systems. Nevertheless during the pilots, some shortcomings emerged that highlighted missing aspects to be considered. Hereafter a list of major issues follows, as result of the pilot findings analysis.

- The current version of the taxonomy specifically addresses needs and functionalities of primary users. The most part of AAL projects focuses on primary users, and often indirectly also on secondary ones. Nevertheless, a there is still a part of AAL projects that specifically focuses on secondary users' needs and the pilot analysis revealed that they could not be properly profiled according to the MAESTRO taxonomy (they do not have necessarily the same problems). More specifically, aspects such as training/support for caregivers is missing in the current version of the MAESTRO taxonomy (some example of projects: POSTHCARD, SUCCESS, SOCIALCARE, iCareCoops.

- Emergency management is not enough considered in the current version of the taxonomy. Some AAL projects offer a specific service in case of emergency which alerts formal and informal caregivers. Related to this, it is worth highlighting the importance to integrate the profiling process with the description of devices included in more complex systems. For example, there is the urgency to consider in the categorization, objects like alarm buttons and fall detectors which are crucial for many systems for the management of emergency situations (i.e. IONIS, SmartBEAT). In the current MAESTRO taxonomy, they appear only as 
part of alerting ecosystems, we should be able to discriminate down to the components.

- Another aspect which seems not to be addressed enough in the proposed profiling framework concerns the concept of therapy adherence which is somehow linked to the issue already mentioned of daily life monitoring. Considering the frequent presence in older persons of chronic diseases which require long-term therapies, an aspect worth being monitored in the daily routine can be surely the adherence to therapy.

- The monitoring of user's habits can sometimes be exploited by AAL systems for delivering healthy advices through a sort of coaching service (i.e. PersonAAL, Ella4Life). Actually, this service has not been considered in designing the MAESTRO profiling framework. In order to overcome this limit, an improvement should be done to the framework by including among possible delivered services those of personalized advices delivering.

Beside these punctual comments, it is important to highlight that the proposed framework mainly focused on needs related to body and mental functioning, instrumental activities of daily life and domestic environmental issues. Considering the global view of AAL program on Active Ageing, there are AAL projects which intend this concept in its broadest sense, and consider the older persons as active members of society.

More specifically, the call 6 focused on "ICT-based Solutions for Supporting Occupation in Life of Older Adults". Those projects resulted difficult to profile through the MAESTRO framework considering the different environmental context intended for application.

\section{Conclusions and Final Remarks}

The paper presents the MAESTRO project providing a brief description of its objectives and an overview of the main components and services. MAESTRO aims at providing innovative ICT services to bridge the gap between end-users like e.g., seniors or caregivers and manufacturers of health-related products. It realizes a twofold value chain which supports end-users in finding products suited for their needs and supports manufacturers that receive feedbacks about the qualities of their products

The paper specifically focuses on the profiling capabilities of MAESTRO and the developed recommendation system services developed to rank products according to the specific health-related needs of end-users that interact with the system. In this way, MAESTRO can facilitate end-users in finding products that best fit their capabilities and health condition.

User and product profiles are built and processes leveraging an internal taxonomy based on the International Classification of Functioning, Disability and Health (ICF) proposed by the World Health Organization (WHO). The ICF model is well 
suited for the objectives of the project because it provides a multi-dimensional classification schema which can be then used to characterize end-users and products according to different correlated perspectives.

Finally, the paper briefly shows an experimental evaluation of the classification capabilities of MAESTRO taking into account AAL projects. It clearly emerged from the pilot results the good capability of the MAESTRO taxonomy of capturing a considerable number of nuances representing the complex and interrelated factors affecting Active Ageing. The choice of relying on the multidimensional ICF classification has resulted excellent in order to identify and classify the end users' needs. Nevertheless, since the ICF focused on individual dimensions, it somehow fails to capture those networking aspects which characterize the AAL world.

In fact, the secondary users point of view was not directly addressed by the Profiling Tool. Additionally, while the efficacy of describing single aspects influencing Active Ageing has been proven, more difficulty emerged in highlighting those aspects resulting from aggregated factors, like for example the daily routine. It represents an aggregation of different dimensions already investigated by the Profiling Tool, but which need additional crucial and high leve reasoning could not easily grasped through this profiling process.

Starting from the current version of the Profiling Tool, and taking into account these few considerations, the commercial version of the Profiling Tool will needs some integrations in order to fully embrace the complexity of the offers from the AAL world.

\section{References}

[Bechhofer, 2009] Bechhofer, S. (2009). OWL: Web Ontology Language, pages 2008-2009. Springer US, Boston, MA.

[Gangemi et al., 2002] Gangemi, A., Guarino, N., Masolo, C., Oltramari, A., and Schneider, L. (2002). Sweetening ontologies with dolce. In Gómez-Pérez, A. and Benjamins, V. R., editors, Knowledge Engineering and Knowledge Management: Ontologies and the Semantic Web, pages 166-181, Berlin, Heidelberg. Springer Berlin Heidelberg.

[Organization et al., 2001] Organization, W. H. et al. (2001). International classification of functioning, disability and health: ICF. Geneva: World Health Organization. 